\title{
Characterizing Heat Release Rates Using an Inverse Fire Modeling Technique
}

\author{
Kristopher J. Overholt and Ofodike A. Ezekoye*, Department of Mechanical \\ Engineering, The University of Texas at Austin, Austin, TX 78712, USA
}

Received: 29 April 2011/Accepted: 26 December 2011

\begin{abstract}
A ubiquitous source of uncertainty in fire modeling is specifying the proper heat release rate (HRR) for the fuel packages of interest. An inverse HRR calculation method is presented to determine an inverse HRR solution that satisfies measured temperature data. The methodology uses a predictor-corrected method and the Consolidated Model of Fire and Smoke Transport (CFAST) zone model to calculate hot gas layer (HGL) temperatures in single compartment configurations. The inverse method runs at super-real-time speeds while calculating an inverse HRR solution that reasonably matches the original HRR curve. Examples of the inverse method are demonstrated by using a multiple step HRR case, complex HRR curves, experimental temperature data with a constant HRR, and a case with an experimentally measured HRR. In principle, the methodology can be applied using any reasonably accurate fire model to invert for the HRR.
\end{abstract}

Keywords: Compartment fires, Fire growth, Fire modeling, Heat release rate,

Inverse fire modeling problems

\section{Introduction}

Currently, the use of fire models in scenarios involving firefighter injuries, line-ofduty deaths, or forensic applications requires a tedious and manual iterative process of modifying the input parameters to create the desired or expected results from a zone or field fire model and comparing the results to a timeline of observations. This process can result in significant errors or nonphysical results from fire models and might not include a sufficiently wide range of conditions that adequately describe the fire effects or fire behavior for a given scenario.

Previous work by Jahn et al. [1] has demonstrated a method to forecast the fire size in an enclosure using sensor-driven inputs. That study used real-time sensor data (e.g., heat detectors, smoke detectors) to steer a fire model and account for changes in the environment of a fire scenario. The goal of that study was to use information from the evolving fire scenario to accelerate model predictions. A study by Cowlard et al. [2] describes the process of using real-time sensor data to assist firefighting operations through the use of high performance computers running numerous fire simulations in parallel and fetching pre-computed scenarios. That study also demonstrated the sensitivity of the model results to the input

\footnotetext{
* Correspondence should be addressed to: Ofodike A. Ezekoye, E-mail: dezekoye@mail.utexas.edu
} 
parameters and how sensor data could be used to steer and correct the simulations.

Additional studies have been performed on sensor-driven fire simulations to determine the location and size of the fire. A paper by Davis and Forney [3] outlines a process for using correlations and zone models as a sensor-driven zone model. A study by Koo et al. [4] used a sensor-driven steering method that performed at super-real-time speeds using high performance computing resources with the ability to run 1,000 scenarios per minute. A study by Richards et al. [5] used transient temperature data from ceiling sensors to determine the heat release rate (HRR) and location of fires in large-scale compartments, but the inverse HRR solution had an error of $300 \%$ to $500 \%$ of the measured HRR. Studies by Neviackas [6], Neviackas and Trouvé [7], and Leblanc and Trouvé [8] used hot gas layer (HGL) temperatures in an enclosure (single and multiple compartments) and a genetic algorithm to search for an average inverse HRR. The genetic algorithm required multiple hours of runtime, and the solution was limited to a constant, time-averaged HRR. In general, the approaches used in these studies are infeasible for general applications because of the amount of computational expenditure required or the inaccuracy of their inversions. However, the need for such inversion capability is evident. The focus of this study is to develop a quick, inexpensive method to compute transient HRR data using known temperature data in an enclosure.

A study by Lee and Lee [9] demonstrated the use of a sequential inverse method to determine the size and location of a compartment fire. In that study, the HRR in a compartment was calculated sequentially by using a discretized form of Alpert's correlation [10] for gas temperatures in ceiling jet flows. The results exhibit a large amount of noise $( \pm 100 \%$ error in the resulting HRR solution), and the correlations apply only to a limited scope of physical scenarios. However, these types of correlations can still be useful in the predictor step of an inverse HRR methodology, which was implemented in the inverse method in this paper and is described in the following section.

An inverse recovery methodology is presented that uses a fire model to search for a HRR that satisfies sampled temperature data in an enclosure. The Consolidated Model of Fire and Smoke Transport (CFAST) zone model [11], which is maintained by the National Institute of Standards and Technology, was used to reconstruct a time-varying inverse HRR solution.

First, a simple case with step increments in the HRR and cases with various complex HRR curves are used to demonstrate the inverse solution method. Then, temperatures from experimental enclosure fire tests are used to determine an inverse HRR solution. Finally, a case with an experimentally measured HRR are used to demonstrate the robustness and accuracy of the method for the calculation of transient HRR solutions.

\section{Inverse Heat Release Rate Solution Methodology}

For a given HGL time-temperature curve, numerous inverse solutions exist that can satisfy the input conditions. One approximation for a HRR solution that 
satisfies the time-temperature curve can be expressed by parameterizing the HRR using a piecewise linear function, as shown in Equation 1.

$$
\dot{Q}(t)=\dot{Q}_{i}\left(\frac{t-t_{i+1}}{t_{i}-t_{i+1}}\right)+\dot{Q}_{i+1}\left(\frac{t-t_{i}}{t_{i+1}-t_{i}}\right),
$$

where $\dot{Q}(t)$ is the HRR $(\mathrm{kW})$, and $\dot{Q}_{i}$ are the calculated HRR values at each time $t_{i}$ that the temperature data are sampled. Mathematically, the problem can be cast as a least squares problem in which the relative error $S(\dot{\mathbf{Q}})$ between the measured and predicted temperatures is minimized, as shown in Equation 2.

$$
S(\dot{\mathbf{Q}})=\frac{\sum_{i=0}^{n}\left(Y_{i}-T_{i}(\dot{\mathbf{Q}})\right)^{2}}{\sum_{i=0}^{n}\left(Y_{i}\right)^{2}},
$$

where $Y_{i}$ are the measured temperatures at time $i, n$ is the number of time samples, and $T_{i}(\dot{\mathbf{Q}})$ are the estimated temperatures found from the direct solution of the problem using some proposed time evolution of $\mathbf{Q}$.

With gradient information (i.e., a sensitivity or Jacobian matrix) on the effects of the transient heat release rate $\dot{\mathbf{Q}}$ on the estimated temperatures, various solution techniques are available. In this optimization problem, the vector of a single variable (HRR) is sought. Note that the vector of the HRR contains the transient HRR over times in which temperature data are available. A predictor-corrector method was used with a set of specified input times and corresponding HGL temperatures. Figure 1 shows a flow diagram of this iterative HRR search procedure. A simple iterative procedure, as described by Özisik and Oranlde [12], was used to obtain the vector of unknown parameters, as shown in Equation 3.

$$
\dot{\mathbf{Q}}^{k+1}=\dot{\mathbf{Q}}^{k}+\mathbf{J}^{-1}\left(\mathbf{Y}-\mathbf{T}\left(\dot{\mathbf{Q}}^{k}\right)\right)
$$

where $\left(\mathbf{Y}-\mathbf{T}\left(\dot{\mathbf{Q}}^{k}\right)\right)=\Delta \mathbf{T}$ is the difference between the measured and predicted temperatures.

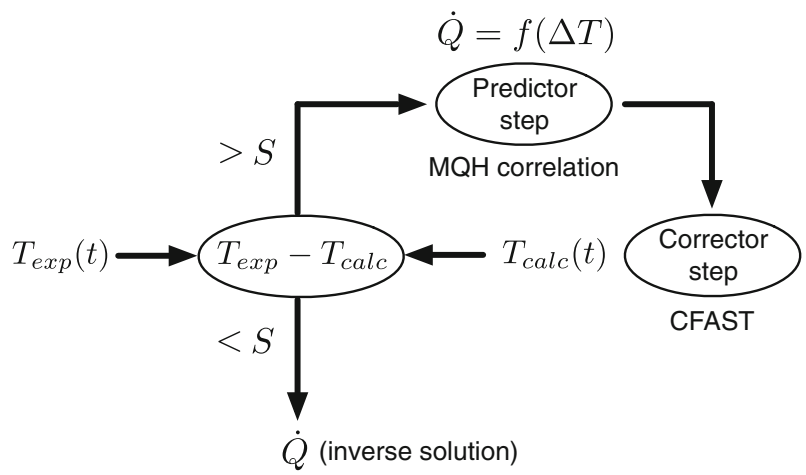

Figure 1. Flowchart illustrating the inverse HRR search procedure. 
The inverse of the sensitivity coefficient $\left(\mathbf{J}^{-1}\right.$ in Equation 3$)$ in these types of problems is typically not known. The temperature sensitivity to HRR is generally a complex relationship that depends on the full vector of HRRs. However, a linear inverse problem that is easily invertible is available by using an analytical correlation by McCaffrey, Quintiere, and Harkleroad $(\mathrm{MQH})$ [13, 14], which is shown in Equation 4. This physical correlation was used to compute the required change in the HRR $(\Delta \dot{\mathbf{Q}})$ based on the difference between the measured and predicted temperatures $(\Delta \mathbf{T})$.

$$
\Delta T_{g}=6.85\left(\frac{\dot{Q}^{2}}{A_{o} \sqrt{H_{o}} h_{k} A_{T}}\right)^{1 / 3},
$$

where $\Delta T_{g}$ is the change in the HGL temperature $\left({ }^{\circ} \mathrm{C}\right), \dot{Q}$ is the HRR $(\mathrm{kW}), A_{o}$ is the ventilation area $\left(\mathrm{m}^{2}\right), H_{o}$ is the ventilation height $(\mathrm{m}), h_{k}$ is the effective heat transfer coefficient of the boundaries $\left(\mathrm{W} / \mathrm{m}^{2}-\mathrm{K}\right)$, and $A_{T}$ is the boundary surface area $\left(\mathrm{m}^{2}\right)$. The ventilation conditions and material properties of the boundary conditions were specified for each case and are described in more detail in the following sections.

The inverse HRR search procedure is summarized in the following steps:

- Step 1: For a temperature difference $(\mathbf{Y}-\mathbf{T})$ between the measured and predicted temperatures, the predictor step computes $\Delta \dot{\mathbf{Q}}$ for all times $t_{i}$ by using the sensitivity, $\mathbf{J}$, (i.e., dT/dQ) found from the $\mathrm{MQH}$ correlation in Equation 4. An intermediate value of $\mathbf{Q}^{k+1}$ based on the MQH correlation is then computed using Equation 3.

- Step 2: For the corrector step, the CFAST model is run with the MQH-derived HRR values $\dot{\mathbf{Q}}^{k+1}$ to generate temperatures $\mathbf{T}^{k+1}$ at the next iteration.

- Step 3: If the error is less than a specified tolerance $\left(S(\dot{\mathbf{Q}}) \leq 1 \times 10^{-3}\right)$, then the resulting $\dot{\mathbf{Q}}$ is returned. Otherwise, Steps 1 and 2 are repeated as the predictorcorrector procedure iterates. The result of the inverse HRR method is a piecewise linear function of HRR versus time, as shown in Equation 1.

The Python programming language, which is a high-level scripting language, was used to generate CFAST input files, run CFAST multiple times while searching for a HRR solution, parse the output from CFAST, and repeat this process to create an inverse HRR solution. This method is demonstrated with various examples in the following sections.

\section{Zone Model Setup}

The zone model, CFAST version 6.2.0, was used in this study. The source code for version 6.2.0 of CFAST was used to compile the CFAST program for the Mac OS X operating system, and the command line binary was controlled by an automatic script rather than using the graphical interface. This approach allowed for the inverse search method to perform efficiently and autonomously. 


\section{Table 1}

Thermal Properties Used for Various Material Boundary Conditions

\begin{tabular}{lccccc}
\hline Material & $k(\mathrm{~W} / \mathrm{m}-\mathrm{K})$ & $c_{p}(\mathrm{~J} / \mathrm{kg}-\mathrm{K})$ & $\rho\left(\mathrm{kg} / \mathrm{m}^{3}\right)$ & $\delta(\mathrm{cm})$ & $\varepsilon(-)$ \\
\hline Gypsum & 0.16 & 900 & 790 & 1.6 & 0.9 \\
Type X gypsum & 0.14 & 900 & 770 & 1.3 & 0.9 \\
Aluminum & 231 & 1,033 & 2,702 & 0.3 & 0.9 \\
Glass fiberboard & 0.04 & 720 & 105 & 8.8 & 0.9 \\
\hline
\end{tabular}

In the CFAST zone model, all of the input parameters (e.g., combustion, solid phase, geometry) were fixed to simplify the search process, and the HRR was the only parameter that was varied. The CFAST zone model was configured as follows: methane was used as the fuel with a heat of combustion of $50 \mathrm{~kJ} / \mathrm{g}$, and the boundary conditions, ambient temperature, ventilation conditions, and compartment geometry were configured for each of the specific cases, which are described in the following sections. The material properties of the boundaries were varied and are shown in Table 1. These boundary conditions were also used in the MQH correlation for the predictor step of the inverse method.

\section{Multiple Step Function Increments in the Heat Release Rate}

First, a simple case is considered in which a fire is simulated with piecewise constant HRRs of $100 \mathrm{~kW}, 200 \mathrm{~kW}$, and $300 \mathrm{~kW}$ at times of $100 \mathrm{~s}, 200 \mathrm{~s}$, and $400 \mathrm{~s}$, respectively. The resulting HGL temperatures from this fire were used as inputs to verify the accuracy of the inverse methodology. The sample resolution of the input temperatures was $10 \mathrm{~s}$. The actual HRR curve is shown as a solid line in Figure $2 b$, and the resulting temperatures (which were inputs to the inverse method) are shown in Figure 2a as points.

Following the procedure described in the previous section, beginning at time zero, the predictor step computes a HRR that satisfies the first input temperature point at the first sample time (i.e., $10 \mathrm{~s}$ ). Next, the predictor step computes a HRR that satisfies the temperature condition at $20 \mathrm{~s}$. This process continues until all of the time-temperature points have associated HRRs. Then, the corrector step involves running the CFAST model to compute the resulting HGL temperatures, and the new error between the measured and the predicted temperatures is calculated. This process continues until a complete inverse HRR solution curve is determined, which is shown as a dashed line in Figure $2 b$. Note that the method overpredicts the HRR near sudden step changes in the HRR. In actual experiments, the HRR is not likely to increase instantaneously, as indicated by the experimentally measured temperatures shown in the following sections. The relative error, as defined by Peacock et al. [15], of the resulting inverse HRR solution is 0.08 in which the relative error is defined as shown in Equation 5. 
(a)

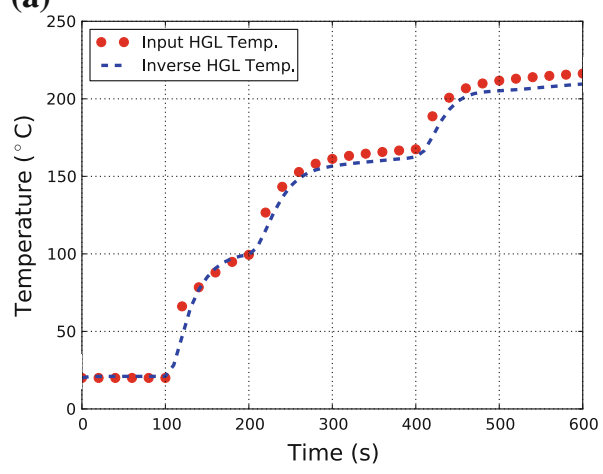

(b)

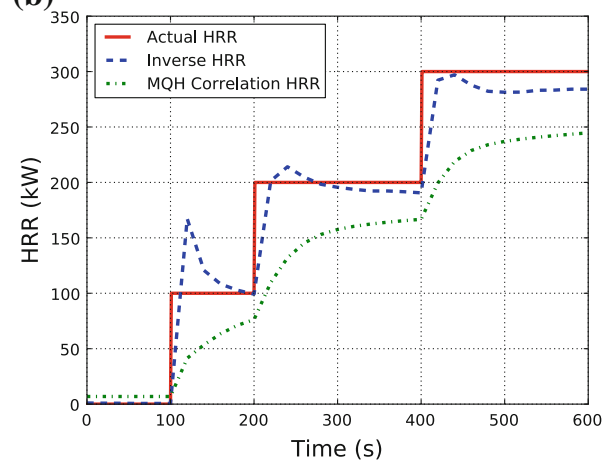

Figure 2. Multiple step function HRR case: (a) HGL temperafures from CFAST (points) and inverse method (dashed line); (b) Inverse HRR curve (dashed line) compared to the actual HRR curve (solid line).

$$
\frac{\|\dot{\mathbf{Q}}-\hat{\mathbf{Q}}\|}{\|\dot{\mathbf{Q}}\|}=\sqrt{\frac{\sum_{i=0}^{n}\left(\dot{Q}_{i}-\hat{Q}_{i}\right)^{2}}{\sum_{i=0}^{n}\left(\dot{Q}_{i}\right)^{2}}},
$$

where $\dot{Q}$ are the actual HRR values and $\hat{Q}$ are the inverse HRR values.

For comparison, the MQH correlation was also used with the same inputs and boundary conditions as the inverse HRR method. Equation 4 was used to compute the HRR at each time step, and the results are shown in Figure $2 b$ as a dash-dot line, which has a relative error of 0.25 . Although the inverse method is a better approximation to the actual HRR, the MQH correlation is still useful in the predictor step of the inverse HRR method, as described in the previous section. By using the predictor-corrector method, this inverse HRR method can be extended to problems that exceed the limitations of the existing correlations by using physics-based models such as CFAST or Fire Dynamics Simulator (FDS) [16].

To demonstrate the sensitivity of the inverse HRR solution to different boundary conditions, two additional cases were run to represent the upper and lower limits for boundary conditions: one case with aluminum boundaries, and one case with glass fiberboard boundaries. The thermal properties for these materials are shown in Table 1. The resulting inverse HRR solutions (Figure 3 ) have a relative error of 0.09 for aluminum and 0.23 for glass fiberboard compared to a relative error of 0.08 for gypsum.

To demonstrate the sensitivity of the inverse HRR solution to noise in the input temperature data, three cases were run with a $5 \%, 10 \%$, and $15 \%$ level of noise in the original input temperature data from CFAST. The noise was applied to the input temperature data assuming a uniform density function centered between $T+\Delta T$ and $T-\Delta T$ (where $\Delta T / T_{\text {mean }}$ is specified for $5 \%, 10 \%$, or $15 \%$ ) using the method shown in Equation 6. 


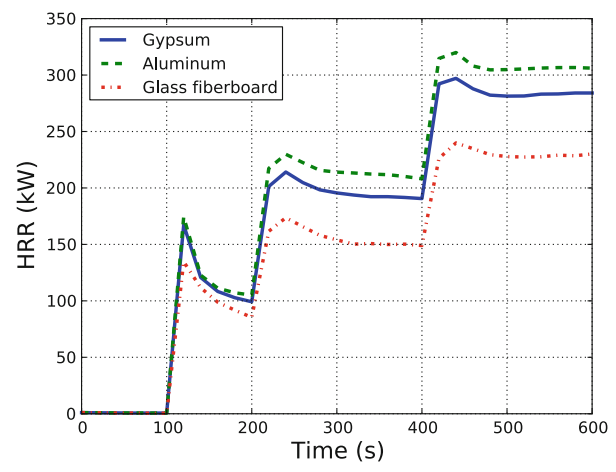

Figure 3. Sensitivity of inverse HRR solution to various material boundary conditions.
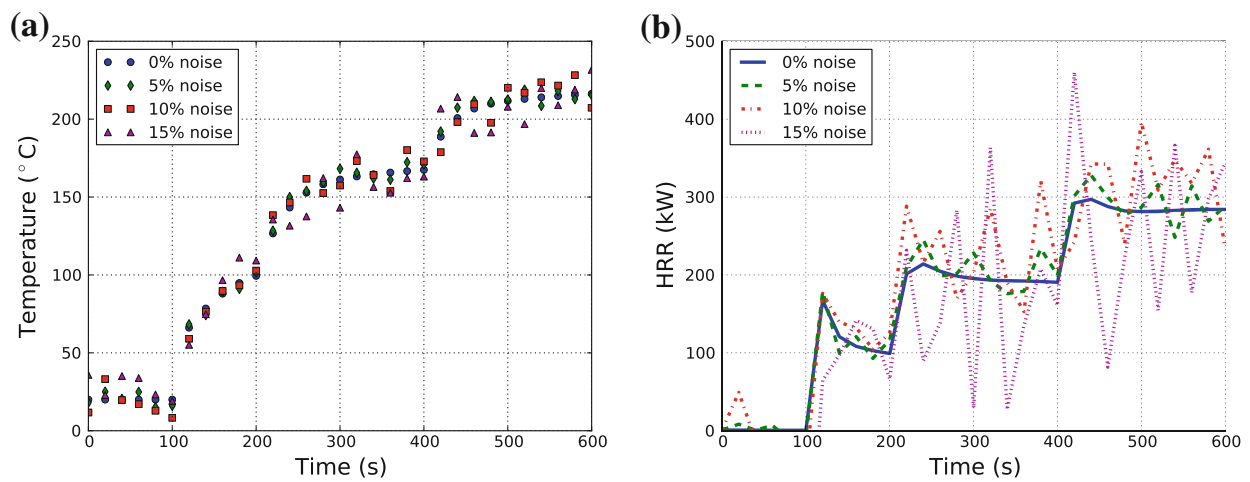

Figure 4. Sensitivity of inverse HRR solution to various levels of randomly perturbed input temperature data: (a) Perturbed HGL temperafures (points) for various levels of noise; (b) Inverse HRR curves for various levels of noise.

$$
T_{i, \text { noise }}(t)=T_{i}(t)+\left(F(T)-\frac{1}{2}\right)\left(\frac{\Delta T}{T_{\text {mean }}}\right),
$$

where $T_{i}(t)$ is the original temperature, $F(T)$ is the cumulative distribution function and is a random number between 0 and 1 , and $T_{\text {mean }}$ is the mean value of the input temperature data.

After the original temperature data were perturbed and a set of $T_{i, n o i s e}(t)$ input temperatures was obtained (Figure 4a), the inverse HRR method was used to determine a HRR solution. The resulting three inverse solutions with different levels of noise are shown in Figure $4 \mathrm{~b}$, where the solid line represents the original inverse HRR solution, the dashed line represents $5 \%$ noise in the temperature data (relative error of 0.13 ), the dash-dot line represents $10 \%$ noise (relative error 


\section{Table 2}

Maximum Change in Temperature and Inverse HRR for Various Amounts of Noise

\begin{tabular}{lcc}
\hline Amount of noise $(\%)$ & Max. $\Delta T\left({ }^{\circ} \mathrm{C}\right)$ & Max. $\Delta Q(\mathrm{~kW})$ \\
\hline 5 & 6 & 42 \\
10 & 13 & 129 \\
15 & 18 & 228 \\
\hline
\end{tabular}

of 0.22 ), and the dotted line represents $15 \%$ noise (relative error of 0.43 ). The resulting maximum change in temperature and inverse HRR for the various amounts of noise are summarized in Table 2.

\section{Complex Heat Release Rate Curves}

To evaluate the ability of the inverse method to determine a solution for complex HRR curves, three example HRR curves from CFAST were used. The original HRR was input into an initial CFAST run to generate synthetic temperature data, and the resulting HGL temperatures were used as inputs for the inverse method to recover the original CFAST HRR curve. The sample resolution of the input HGL temperature data was $10 \mathrm{~s}$ for all of the cases.

For simplicity, the gas phase combustion parameters were the same as in the previous section (i.e., methane with a heat of combustion of $50 \mathrm{MJ} / \mathrm{kg}$ ); therefore, the HRR curve was the only independent search parameter. The enclosure dimensions were the same for all of the cases $(6.1 \mathrm{~m} \times 4.9 \mathrm{~m} \times 2.4 \mathrm{~m}$ enclosure).

Figures 5a, 6a, and $7 \mathrm{a}$ show the synthetic HGL temperature versus time (points) compared the final HGL temperature versus time (dashed line) from the
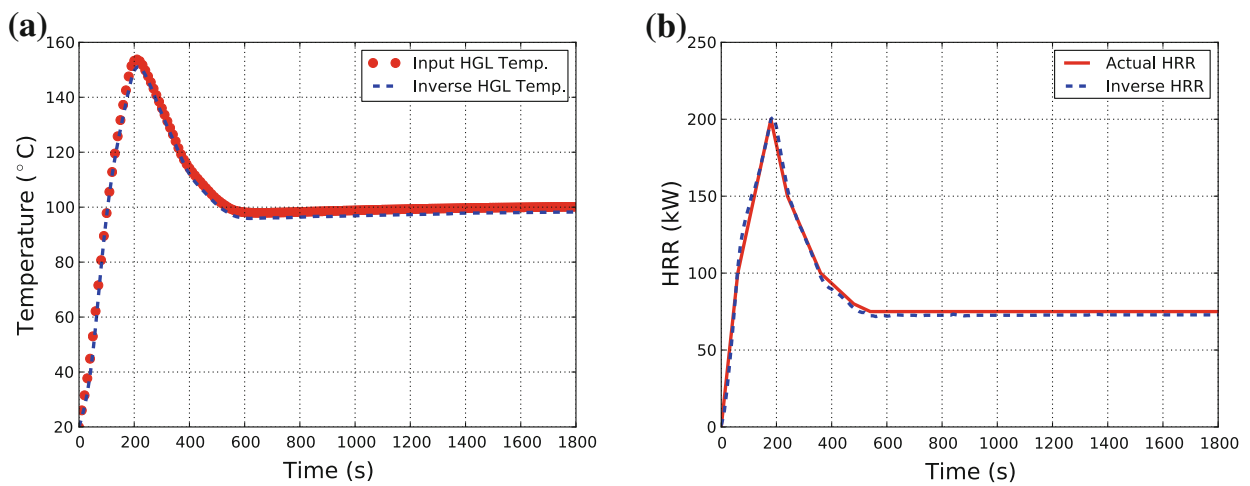

Figure 5. Results from the simple burner HRR case: (a) HCL temperatures from CFAST (points) and inverse method (dashed line); (b) Inverse HRR curve (dashed line) compared to actual HRR curve (solid line). 

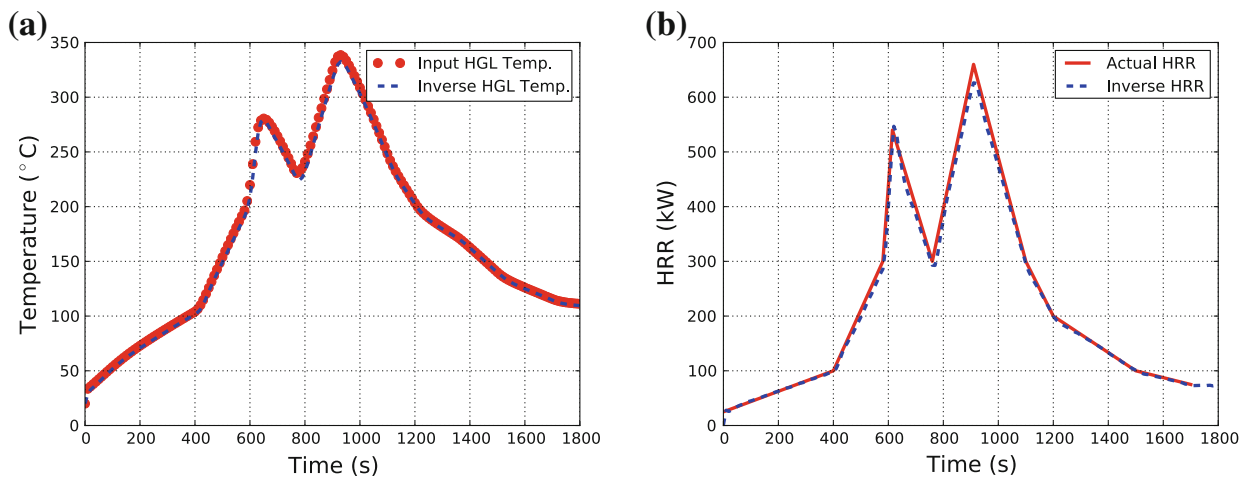

Figure 6. Results from the mattress HRR case: (a) HCL temperatures from CFAST (points) and inverse method (dashed line); (b) Inverse HRR curve (dashed line) compared to actual HRR curve (solid line).

(a)

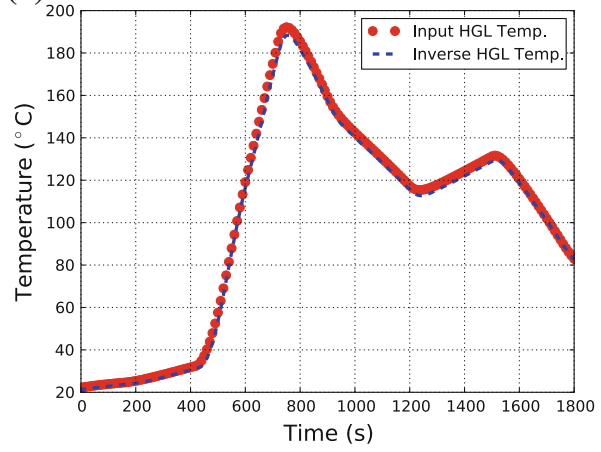

(b)

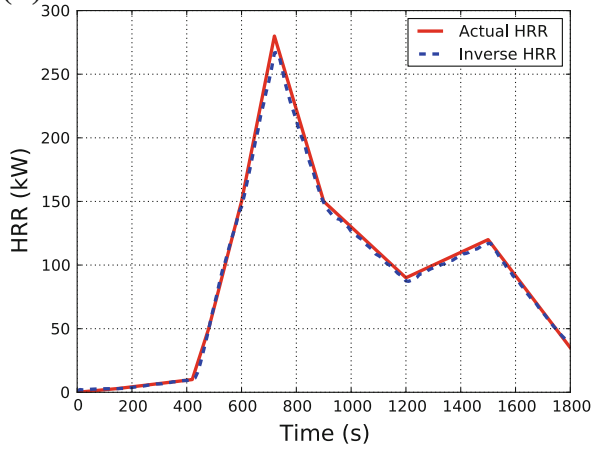

Figure 7. Results from the television HRR case: (a) HCL temperatures from CFAST (points) and inverse method (dashed line); (b) Inverse HRR curve (dashed line) compared to actual HRR curve (solid line).

inverse method for a simple burner case, a mattress and boxspring case, and a television set case, respectively. In Figs. 5b, 6b, and 7b, the original HRR curve is shown as a solid line, and the inverse HRR solution is shown as a dashed line. For all of the cases, the inverse HRR curves are in good agreement with the actual HRR curves. The relative error of the inverse HRR solutions for all three cases is 0.04 .

\section{Experimentally Measured Compartment Temperature Data}

The inverse method was applied to various scenarios involving actual fire conditions by using experimentally measured temperatures from enclosure fire 
experiments as inputs to the inverse method. The experimental setup, input values, and resulting inverse HRR solutions are described in the following sections.

\subsection{Steckler Compartment Data}

The experimental steady-state compartment temperatures from 11 tests with various ventilation areas from the Steckler compartment fire data [17] were used as inputs to the inverse method.

For each Steckler compartment test, the ventilation area and ambient temperature were input into the CFAST simulations, and the average HGL temperatures reported from the experiments were used as inputs to the inverse method. The inverse method was then used to determine a steady-state HRR that would result in the HGL temperatures for each test, and the results of the inverse HRR method are shown in Table 3. The results are in good agreement with the experimental compartment data from Steckler and are within $6 \%$ of the experimental HRR value. For the tests considered in this study, Steckler reports a fire size of $62.9 \mathrm{~kW}$ for each test. Assuming a typical rotameter accuracy of $2 \%$ of full scale with a maximum flow rate of $3.2 \times 10^{-3} \mathrm{~m}^{3} / \mathrm{s}$, which corresponds to the largest HRR reported by Steckler $(158 \mathrm{~kW})$, this translates into a HRR uncertainty of $\pm 6 \mathrm{~kW}$ in the case of methane, or an uncertainty of about $10 \%$ for the $62.9 \mathrm{~kW}$ case. Therefore, the results of the inverse HRR method can be considered to be close to or within the uncertainty bounds of the experimental data from Steckler.

\subsection{UT Austin Experimental Data}

The inverse method was then used with transient temperature data from compartment fire experiments that were performed at The University of Texas at Austin in a $6.1 \mathrm{~m} \times 4.9 \mathrm{~m} \times 2.4 \mathrm{~m}$ enclosure [18]. The walls and ceiling of the enclosure were lined with one layer of $1.6 \mathrm{~cm}$ (0.63 in) gypsum wallboard. A schematic of the experimental setup is shown in Figure 8. The experiments used two propane

\section{Table 3}

\section{Error in Inverse HRR Solution Versus Vent Width From the Steckler Experiments}

\begin{tabular}{lccc}
\hline Vent width $(\mathrm{m})$ & Reported HRR $(\mathrm{kW})$ & Inverse HRR $(\mathrm{kW})$ & HRR error $(\%)$ \\
\hline 0.24 & 62.9 & 63.5 & 0.9 \\
0.36 & 62.9 & 62.5 & 0.7 \\
0.49 & 62.9 & 63.1 & 0.3 \\
0.49 & 62.9 & 66.4 & 5.6 \\
0.62 & 62.9 & 61.4 & 2.4 \\
0.74 & 62.9 & 61.4 & 2.4 \\
0.74 & 62.9 & 60.4 & 3.9 \\
0.74 & 62.9 & 61.1 & 2.9 \\
0.74 & 62.9 & 65.8 & 4.7 \\
0.86 & 62.9 & 61.2 & 2.7 \\
0.99 & 62.9 & 59.5 & 5.4 \\
\hline
\end{tabular}




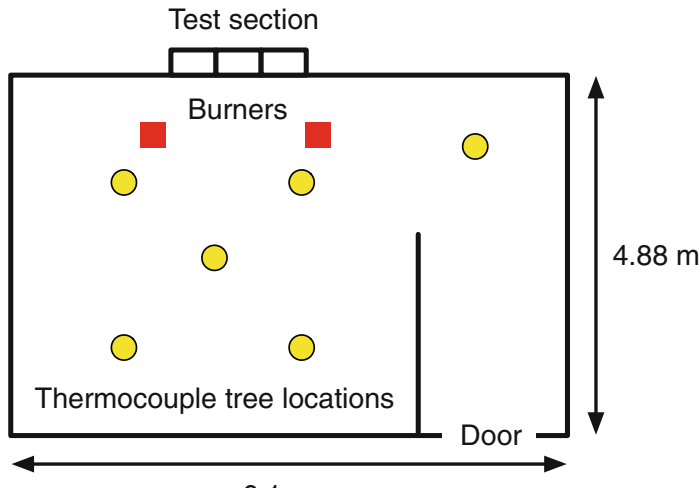

$6.1 \mathrm{~m}$

Figure 8. Experimental setup of the burn structure at The University of Texas at Austin.

(a)

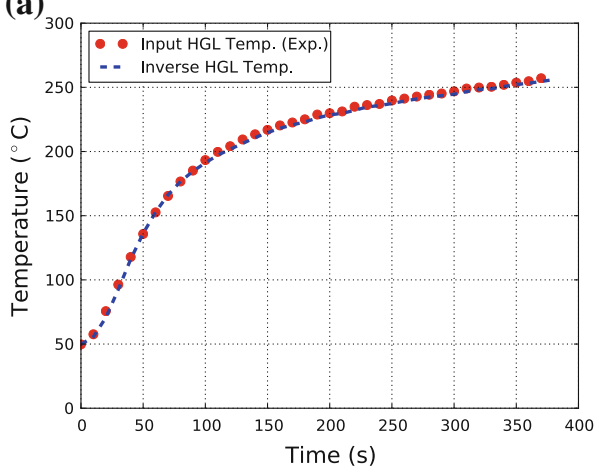

(b)

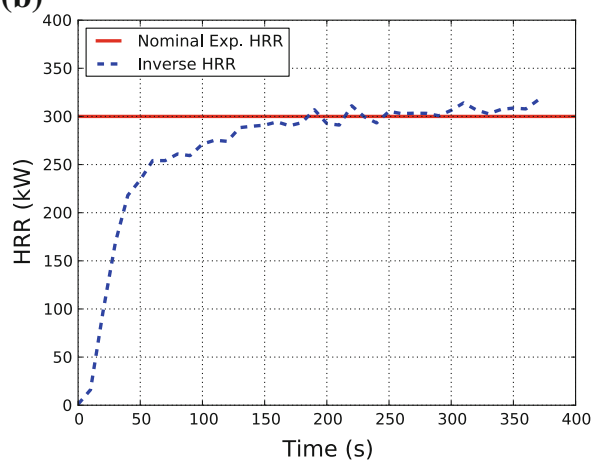

Figure 9. Experimental HRR case with one $300 \mathrm{~kW}$ step: (a) HCL temperatures from CFAST (points) and inverse method (dashed line); (b) HRR curve from inverse search.

burners with total nominal HRRs of $300 \mathrm{~kW}$ and $400 \mathrm{~kW}$, and all doors and vents were closed during the time period shown. The burn structure was instrumented with 32 thermocouples (eight thermocouple trees with four thermocouples each at various heights). The time at which the burners were activated can be considered to be a step function change in the HRR, and the inverse solution is then compared to this step change.

Figure 9a shows the experimentally measured compartment temperature versus time (points) compared to the final HGL temperature versus time (dashed line) from the inverse method. The experimentally measured HGL temperatures (points) shown in Figure 9a represent a spatially averaged temperature over six thermocouple trees using the highest thermocouples $(2.08 \mathrm{~m})$ in the local fire area (the other two thermocouple trees were located behind a wall near the door). This 

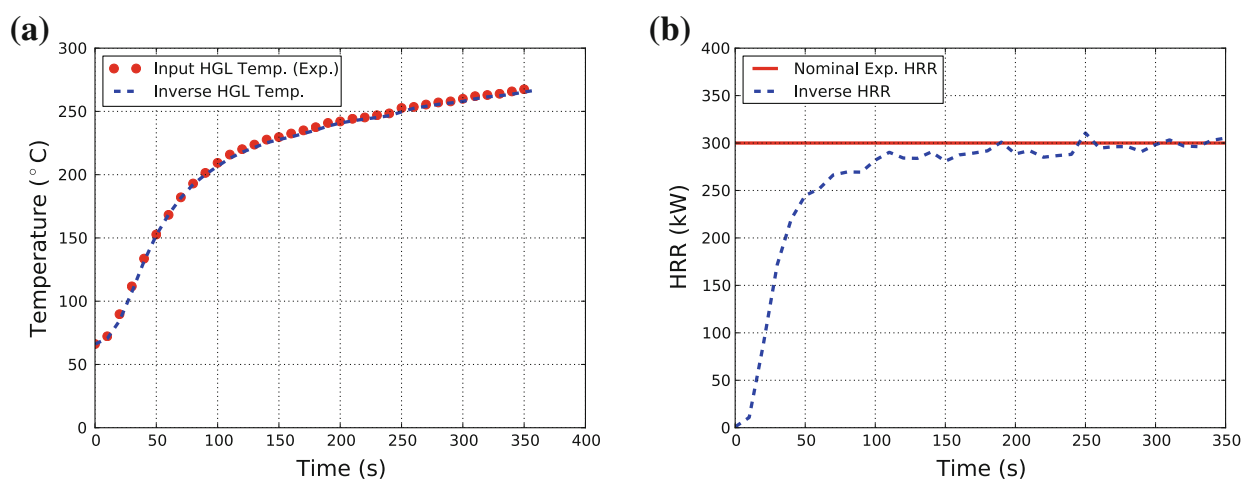

Figure 10. Experimental HRR case with one $300 \mathrm{~kW}$ step: (a) HGL temperatures from CFAST (points) and inverse method (dashed line); (b) HRR curve from inverse search.
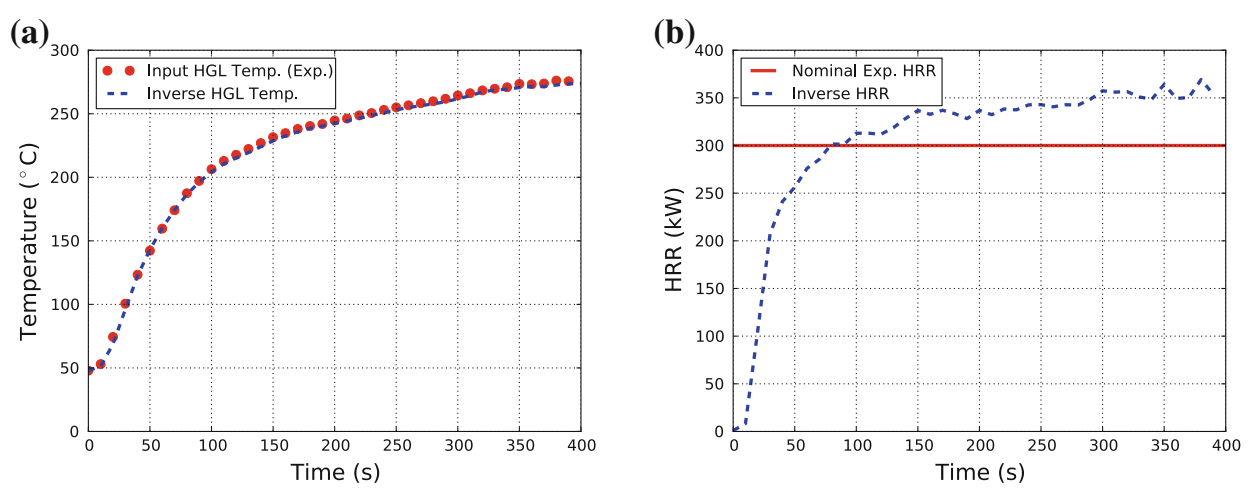

\section{Figure 11. Experimental HRR case with one $300 \mathrm{~kW}$ step: (a) HCL temperatures from CFAST (points) and inverse method (dashed line); (b) HRR curve from inverse search.}

spatially averaged temperature was used as an approximation to the HGL temperature calculated by CFAST. For the inverse HRR method, the sample resolution for the temperature inputs was $10 \mathrm{~s}$. The ambient temperature in the CFAST model was matched to that of the experiments. Figure $9 \mathrm{~b}$ shows the inverse HRR solution (dashed line) compared to the nominal experimental HRR (solid line) based on the fuel mass flow rate to the gas burners.

Figures 10a, 11a, and 12a show the experimentally measured HGL temperature versus time (points) for three additional fire tests compared to the final HGL temperature versus time (dashed line) from the inverse method. Figures 10b, 11b, and $12 \mathrm{~b}$ show the inverse HRR solution (dashed line) compared to the nominal experimental HRR (solid line) based on the fuel mass flow rate to the gas burners. The tests shown in Figs. 9, 10, and 11 had a nominal HRR of $300 \mathrm{~kW}$, whereas the 

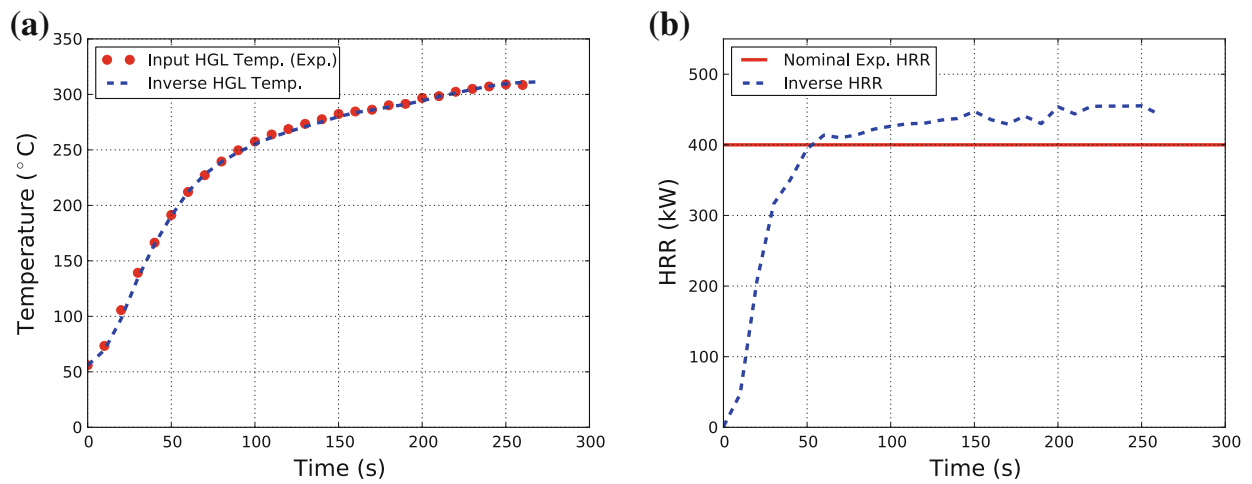

\section{Figure 12. Experimental HRR case with one 400 kW step: (a) HGL temperatures from CFAST (points) and inverse method (dashed line); (b) HRR curve from inverse search.}

test shown in Figure 12 had a nominal HRR of $400 \mathrm{~kW}$. Overall, the uncertainty of $\dot{Q}$ is approximated as $5 \%$ by considering the uncertainty in the rotameter settings. The relative errors between the nominal HRR and inverse HRR solutions are between $7 \%$ and $19 \%$.

\section{Experimentally Measured Heat Release Rate Data}

To compare the inverse HRR solution to an experimentally measured HRR, the inverse method was used with transient temperature data from compartment fire experiments that were conducted at Southwest Research Institute (SwRI) ${ }^{1}$. The experiments were performed in a $4.65 \mathrm{~m} \times 3.43 \mathrm{~m} \times 2.43 \mathrm{~m}$ enclosure with a 2 $\mathrm{m} \times 0.74 \mathrm{~m}$ doorway opening. The walls and ceiling of the enclosure were lined with two layers of $1.3 \mathrm{~cm}(0.5 \mathrm{in})$ type $\mathrm{X}$ gypsum wallboard. Figure 13 shows a diagram of the experimental setup. The compartment was instrumented with 35 thermocouples ( 5 ceiling thermocouples, 16 doorway thermocouples, and 14 thermocouples located in thermocouple trees).

A representative test from the full set of furniture experiments was selected for this paper in which a mockup furniture specimen was burned in the enclosure. In that test, the furniture item was a three-seat sofa with cotton fabric and low density polyurethane foam padding placed on a steel frame. The specimen was ignited on the front using a CAL TB 133 gas burner $(19 \mathrm{~kW})$. The combustion products from the enclosure were collected in a furniture calorimeter hood, and the HRR was measured using oxygen consumption calorimetry. For the inverse HRR method, the sample resolution for the temperature inputs was $10 \mathrm{~s}$. Additionally,

\footnotetext{
${ }^{1}$ This section summarizes partial results from SwRI Project No. 15998. This project was supported by Award No. 2010DN-UX-K221, awarded by the National Institute of Justice, Office of Justice Programs, U.S. Department of Justice. The opinions, findings, and conclusions or recommendations expressed in this paper are those of the author and do not necessarily reflect those of the Department of Justice.
} 


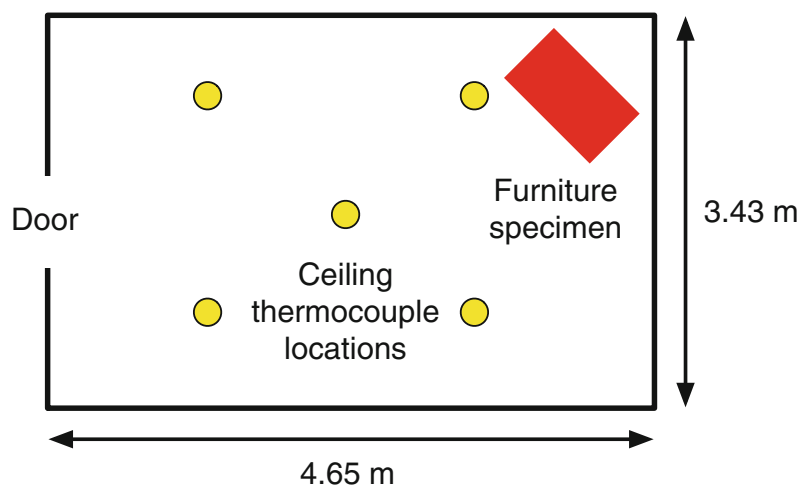

Figure 13. Experimental setup of the furniture testing enclosure at SwRI.
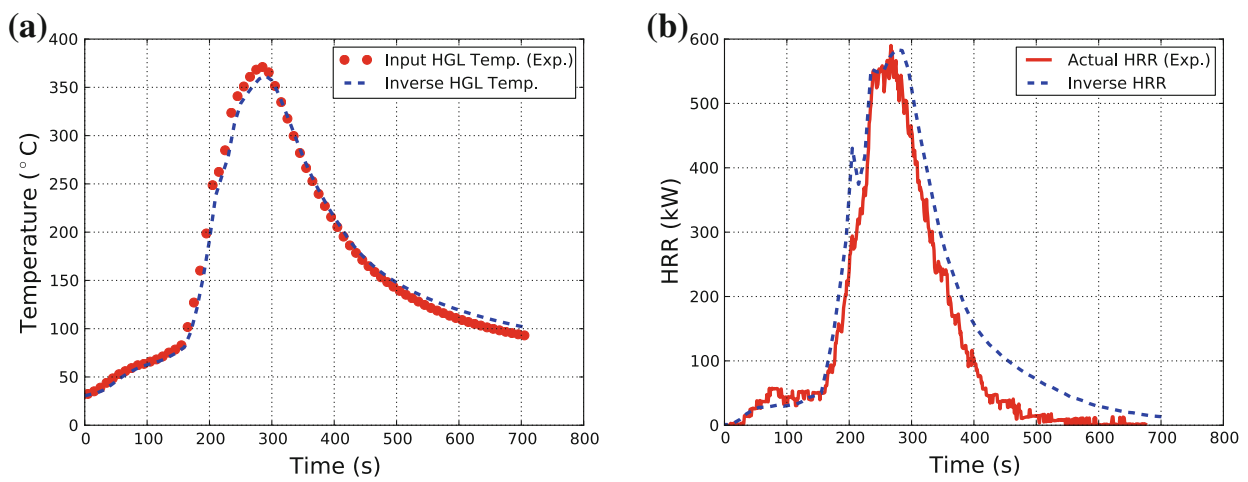

Figure 14. Experimentally measured HRR case: (a) HCL temperatures from CFAST (points) and inverse method (dashed line); (b) Inverse HRR curve (dashed line) compared to the actual HRR curve (solid line).

the ambient temperature in the CFAST model was matched to that of the experiment.

Figure 14a shows the experimentally measured compartment temperatures versus time (points) compared to the final HGL temperature versus time (dashed line) from the inverse method. The input HGL temperatures (points) shown in Figure 14a represent a spatially averaged temperature over the five ceiling thermocouples. This spatially averaged temperature was used as an approximation to the HGL temperature calculated by CFAST. Figure $9 \mathrm{~b}$ shows the experimentally measured HRR (solid line) compared to the inverse HRR solution (dashed line), which has a relative error of 0.24 . 


\section{Fułure Extensions of Inverse Fire Modeling Techniques}

As faster computing resources become more readily available, these methods will become more important in the application of inverse fire modeling problems (IFMP). Additionally, this method can be used to quickly determine a unique HRR curve that corresponds to an observed fire timeline (e.g., time-temperature history, heat flux measurements, fire service events, ventilation events), which describes a complex IFMP scenario. Fire Dynamics Simulator and CFAST models can be used with various time dependent observations such as the time of window breakages, time of ventilation events, amount of smoke from ventilation openings, and time to flashover to better determine an inverse solution by using physical changes in the environment as bounding conditions. Additional measurements from experiments or fire incidents, such as heat fluxes and smoke layer heights, can be used to improve the inverse solution by imposing physical bounds on the inverse solution.

While the CFAST zone model is relatively inexpensive for this inverse HRR method, the results are based upon assumptions and simplifications of the underlying physics. In principle, this inverse method could be used with more complex fire models such as FDS to determine the resulting enclosure conditions (e.g., temperatures, heat fluxes) and further improve the inverse solution. Automated CFAST runs could be used to vary the fire size and location in the enclosure, and the resulting scenario and HRR could then be simulated in FDS to verify the physics with more fidelity. Previous related work has been performed by Hostikka et al. [19] regarding the probabilistic simulation of CFAST using the Monte Carlo method. That study utilized rank order correlations to identify model parameters that significantly affect the results. Because CFAST is computationally inexpensive compared to FDS, the predictor step of the inverse solution could quickly be computed using CFAST, and the results from CFAST could be used to steer subsequent FDS simulations in the corrector step.

\section{Conclusion}

A method for recovering transient HRR based upon measured transient compartment fire temperatures was presented. The inverse method required about 5 to $10 \mathrm{~s}$ of total run time on an Apple Macbook Pro computer with a $2.2 \mathrm{GHz}$ processor to calculate a transient inverse HRR solution for each case; each case required between 10 and 30 CFAST runs for each case. For all of the cases described in this paper, the inverse HRR solution had a relative error between 0.04 and 0.24 compared to the actual HRR. The implementation of the low-order MQH correlation for the predictor step allows for a quick calculation of the update (predictor) step because it has the advantage of being directly invertible for the HRR. Use of the predictor step reduced the total number of computational (CFAST) iterations required to generate an inverse HRR solution that meets the specified convergence criterion.

For the multiple step function increment cases, the inverse solution adequately detected changes in the HRR steps. For the experimental enclosure temperature 
case, the inverse method effectively captured the activation of the gas burners. However, because the HRR was not measured directly, it is difficult to quantify the amount of error in the inverse solution. Qualitatively, this method captured a change in the HRR and exhibits potential for obtaining an inverse solution from these types of scenarios in which the measured HRR is unknown and only temperature data are available. For the complex HRR cases and the experimentally measured HRR case, the inverse method performed well and the inverse HRR solution was in good agreement with the actual HRR, which demonstrates the versatility and accuracy of the inverse HRR method.

One limitation of this methodology is that the material properties of the boundary conditions must be prespecified, and the inverse HRR solution is sensitive to the selection of boundary conditions, as shown in Figure 3. However, in the United States, most of the compartment configurations in which this method can potentially be applied (e.g., residential and commercial occupancies, fire experiments, fire investigations) are limited to certain types of boundary conditions such as gypsum wallboard or similar types of insulating building materials. Thus, it is believed that a computationally inexpensive methodology for the transient HRR solution for such cases is a valid contribution of this study.

\section{Acknowledgments}

This work was funded by the National Institute of Standards and Technology Dept. of Commerce Grant No. 60NANB7D6122.

\section{References}

1. Jahn W, Rein G, Torero J (2011) Forecasting fire growth using an inverse zone modelling approach. Fire Safety J 46(3):81-88

2. Cowlard A, Jahn W, Abecassis-Empis C, Rein G, Torero J (2010) Sensor assisted fire fighting. Fire Technol 46:719-741

3. Davis W, Forney G (2001) A Sensor-driven fire model. National Institute of Standards and Technology Special Publication 965. Gaithersburg, MD, pp 494-505

4. Koo S, Fraser-Mitchell J, Welch S (2010) Sensor-steered fire simulation. Fire Safety J 45(3): 193-205

5. Richards R, Munk B, Plumb O (1997) Fire detection, location and heat release rate through inverse problem solution. Part I: theory. Fire Safety J 28(4):323-350

6. Neviackas A (2007) Inverse fire modeling to estimate the heat release rate of compartment fires. Master's thesis, University of Maryland, College Park

7. Neviackas A, Trouvé A (2007) Sensor-driven inverse zone modeling of enclosure fire dynamics. In: SFPE Professional Development Conference and Exposition. Las Vegas, NV

8. Leblanc M, Trouvé A (2009) Inverse zone modeling of enclosure fire dynamics. In: 6th U.S. Combustion Meeting, Ann Arbor, MI

9. Lee W, Lee S (2005) The estimation of fire location and heat release rate by using sequential inverse method. J Chin Soc Mech Eng 26(1-2):201-207 
10. Alpert R (1972) Calculation of response time of ceiling-mounted fire detectors. Fire Technology 8(3):181-195

11. Peacock R, Jones W, Reneke P, Forney G (2008) CFAST - consolidated model of fire growth and smoke transport (version 6) user's guide. National Institute of Standards and Technology Special Publication 1041. Gaithersburg, MD

12. Özisik M, Orlande H (2000) Inverse heat transfer: fundamentals and applications. Hemisphere Pub, Washington, DC

13. McCaffrey B, Quintiere J, Harkleroad M (1981) Estimating room temperatures and the likelihood of flashover using fire test data correlations. Fire Technol 17(2):98-119

14. Quintiere J (1983) A simple correlation for predicting temperature in a room fire. NBSIR 83-2712, National Bureau of Standards. Washington, DC

15. Peacock R, Reneke P, Davis WD, Jones W (1999) Quantifying fire model evaluation using functional analysis. Fire Safety J 33(3):167-184

16. McGrattan K, McDermott R, Hostikka S, Floyd J (2010) Fire dynamics simulator (version 5) - user's guide. National Institute of Standards and Technology Special Publication 1019-5. Gaithersburg, MD

17. Steckler K, Quintiere J, Rinkinen W (1982) Flow induced by fire in a compartment. In: Symposium (international) on combustion, vol. 19(1). Elsevier, pp 913-920

18. Weinschenk C, Beal C, Ezekoye O (2011) Modeling fan-driven flows for firefighting tactics using simple analytical models and CFD. J Fire Protect Eng 21(2):81-114

19. Hostikka S, Korhonen T, Keski-Rahkonen O (2005) Two-model Monte Carlo simulation of fire scenarios. In: Fire safety science - proceedings of the eighth international symposium, September, pp 18-23 